\title{
Control Release and Diffusion-Reaction Kinetics of Genipin- Eluting Fibers Using an In Vitro Aneurysm Flow Model
}

Emily Augustine, ${ }^{a}$ Puqing Deng, ${ }^{a}$ Chenchen Mou, ${ }^{b}$ Malia Okamura, ${ }^{a}$ Brian Woolley, ${ }^{b, c}$ Michael Horowitz, ${ }^{d}$ Christopher J. Bettinger ${ }^{a, b *}$

${ }^{\text {aDepartment }}$ of Materials Science and Engineering, Carnegie Mellon University, Pittsburgh, PA 15213, USA

bepartment of Biomedical Engineering, Carnegie Mellon University, Pittsburgh, PA 15213, USA

${ }^{\mathrm{c}}$ Department of Chemical Engineering, Carnegie Mellon University, Pittsburgh, PA 15213, USA

dFirst Coast Neurosurgery, 1887 Kingsley Avenue, Suite 1900, Orange Park, FL 32073

*Email: cbetting@andrew.cmu.edu

Pages: 6

Figures: 7

Tables: 0 


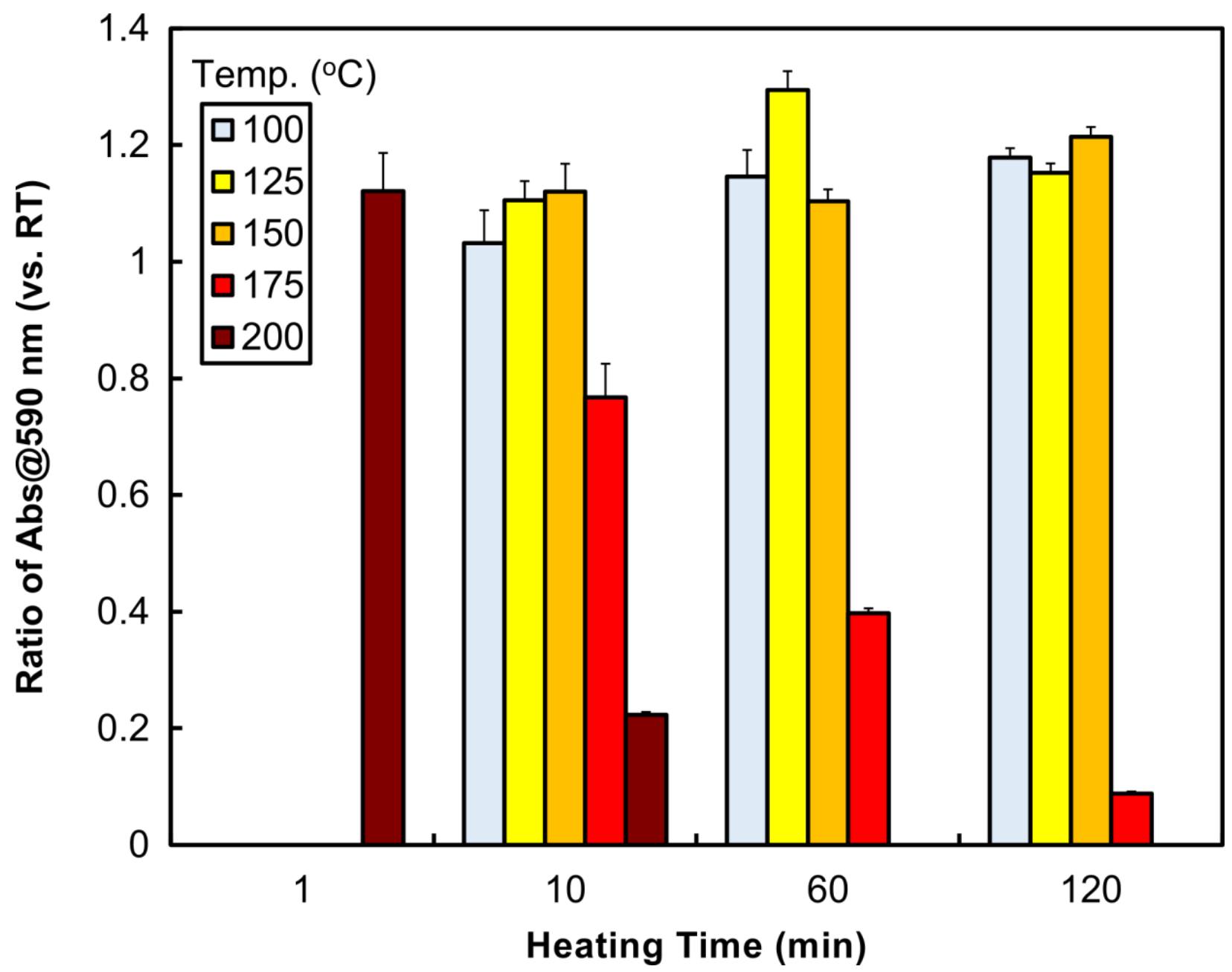

Figure S1. Thermal stability of genipin. Genipin is active after heating for $120 \mathrm{~min}$ at $150{ }^{\circ} \mathrm{C}$. A substantial decrease in activity is observed after heating for $10+$ min at $175^{\circ} \mathrm{C}$ and $200{ }^{\circ} \mathrm{C}$. 
a

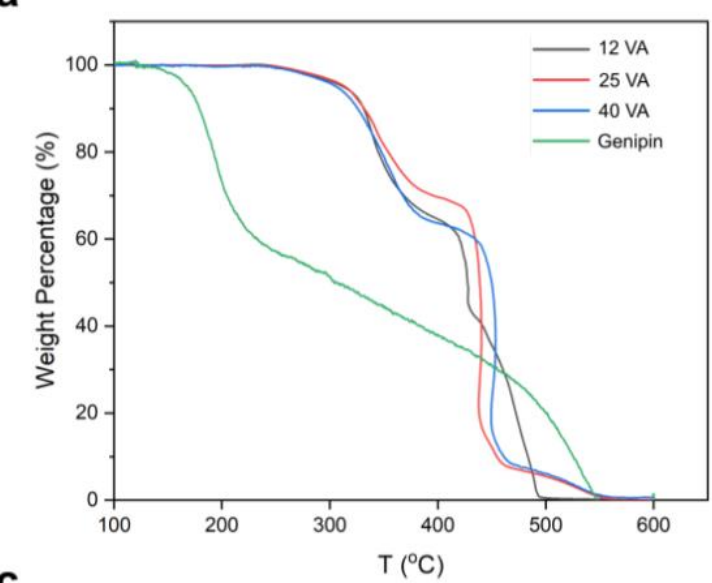

C

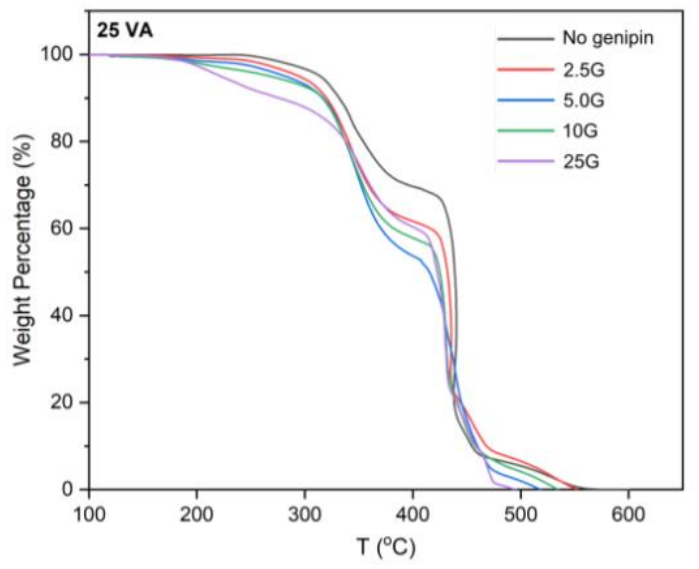

b

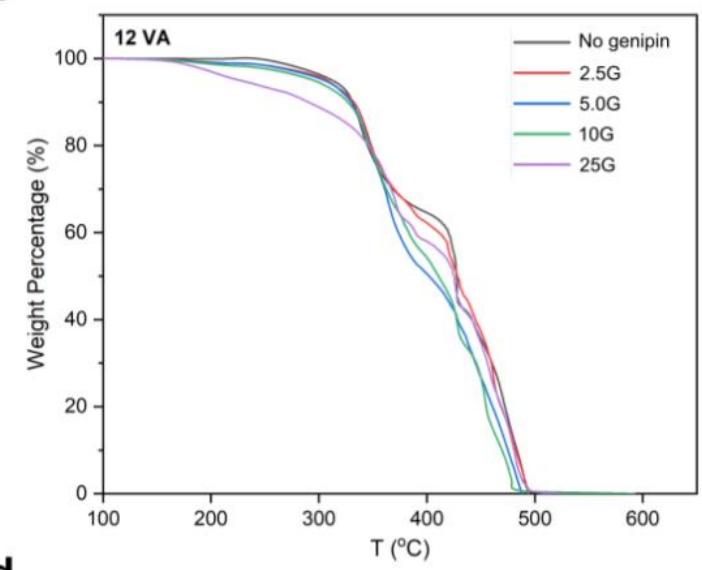

d

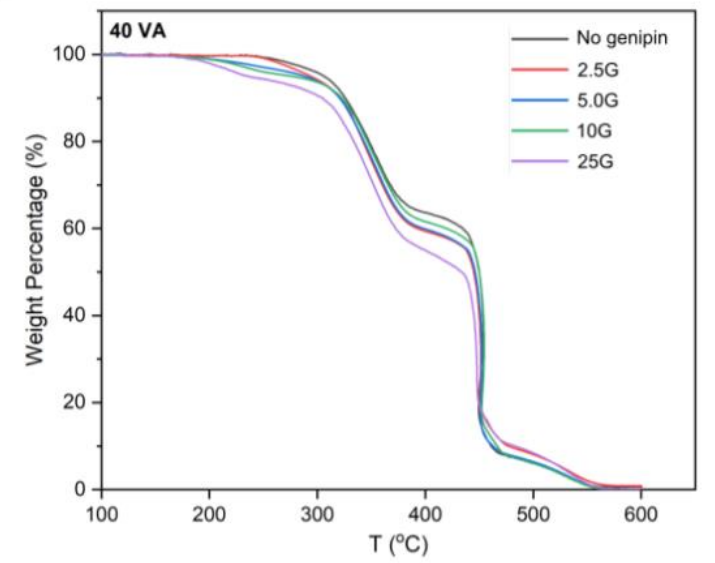

Figure S2. TGA curves of genipin-loaded PEVA fibers. (a) Controls: genipin (green), 12\% vinyl acetate (black), 25\% vinyl acetate (red), 40\% vinyl acetate (blue). PEVA fibers loaded with 2.5, 5, 10 , and $25 \mathrm{wt} \%$ genipin and vinyl acetate concentration of (b) $12 \%$ vinyl acetate, (c) $25 \%$ vinyl acetate, and (d) 40\% vinyl acetate. 

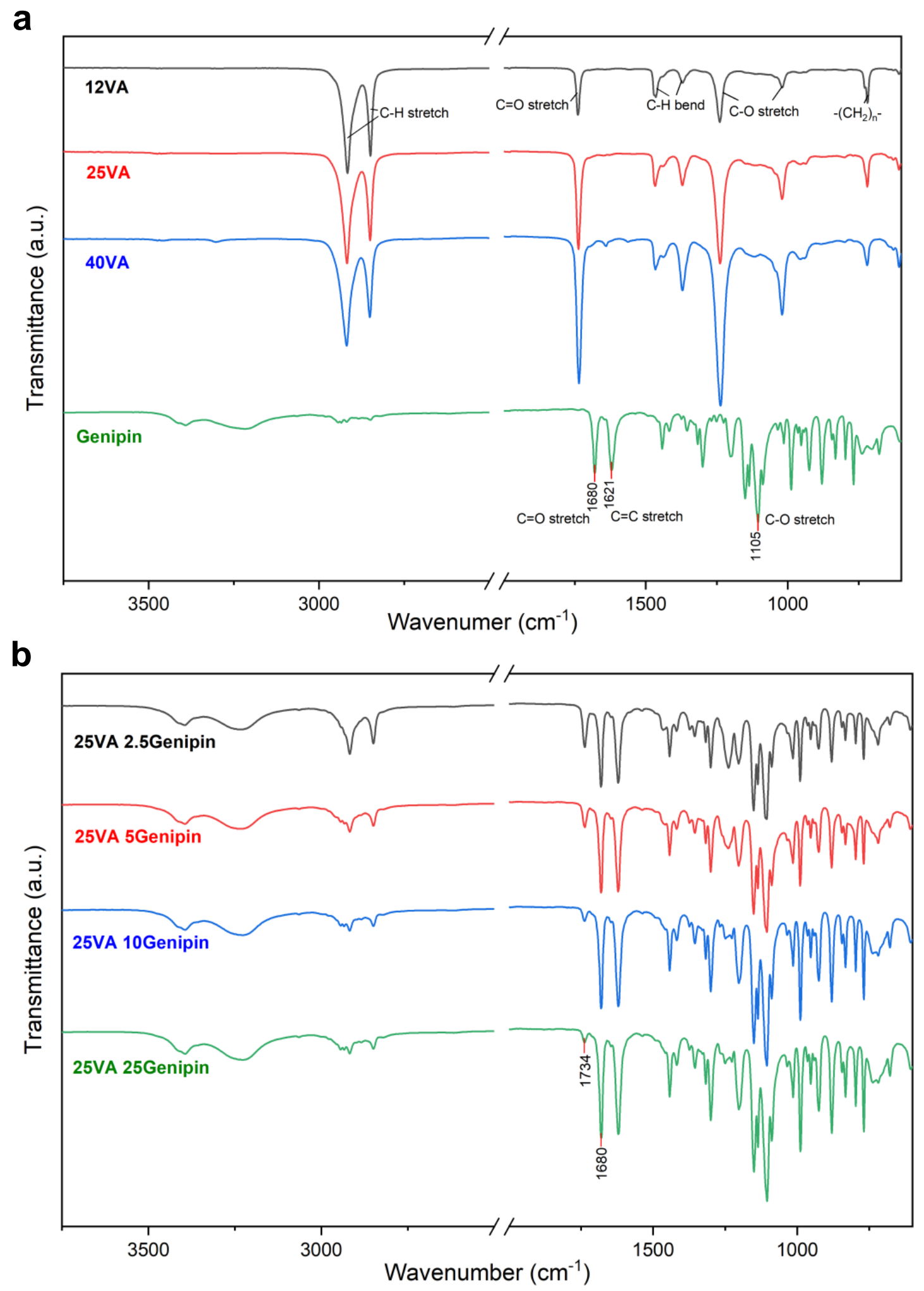

Figure S3. ATR-FTIR spectra. (a) Controls: genipin (green), 12\% vinyl acetate PEVA (black), $25 \%$ vinyl acetate PEVA (red), and 40\% vinyl acetate PEVA (blue). (b) $25 \%$ vinyl acetate PEVA fibers loaded with $2.5,5,10$, and $25 \mathrm{wt} \%$ genipin. 


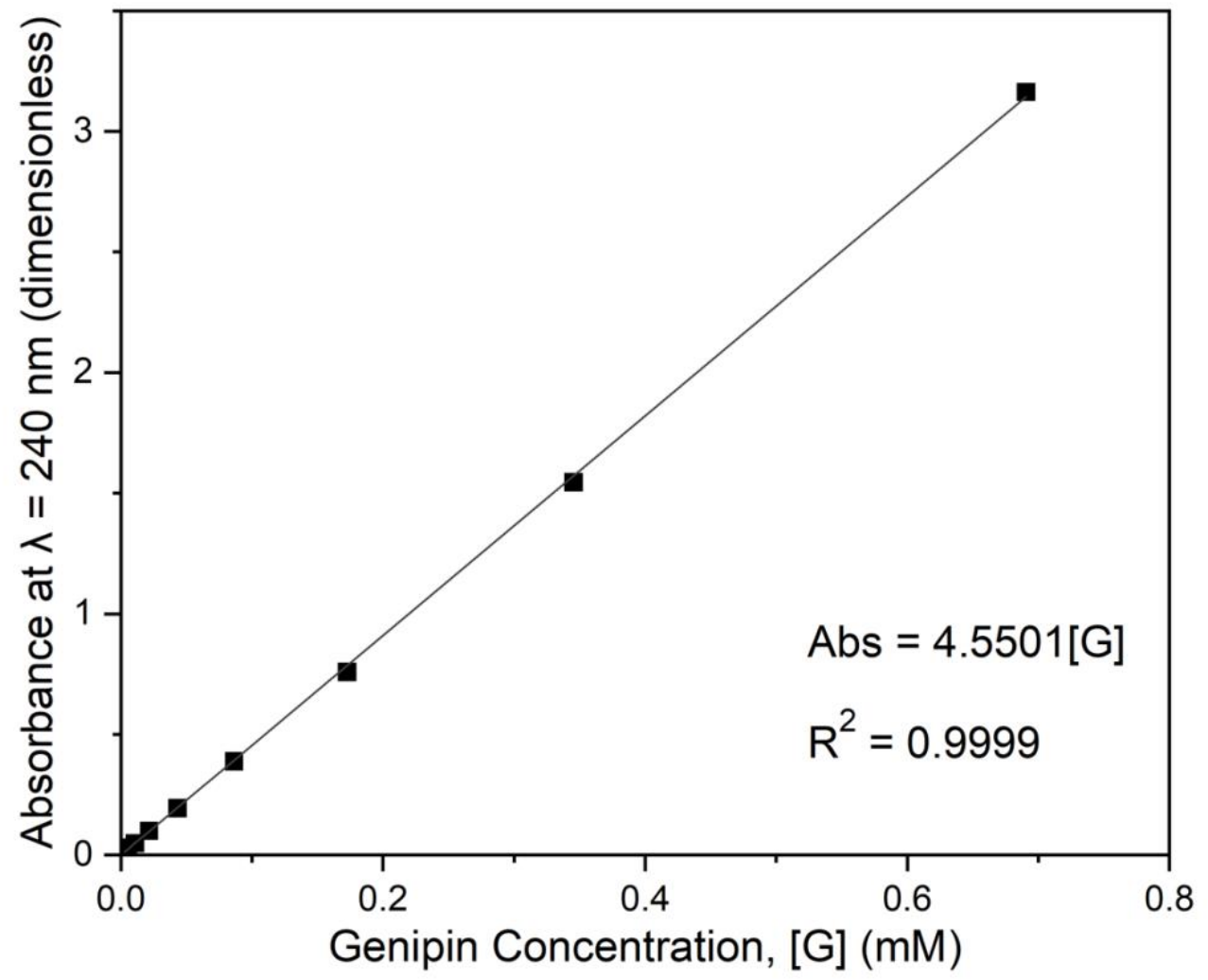

Figure S4. UV-Vis standard curve of genipin concentration versus absorbance at $\lambda$ Abs $=240 \mathrm{~nm}$. 

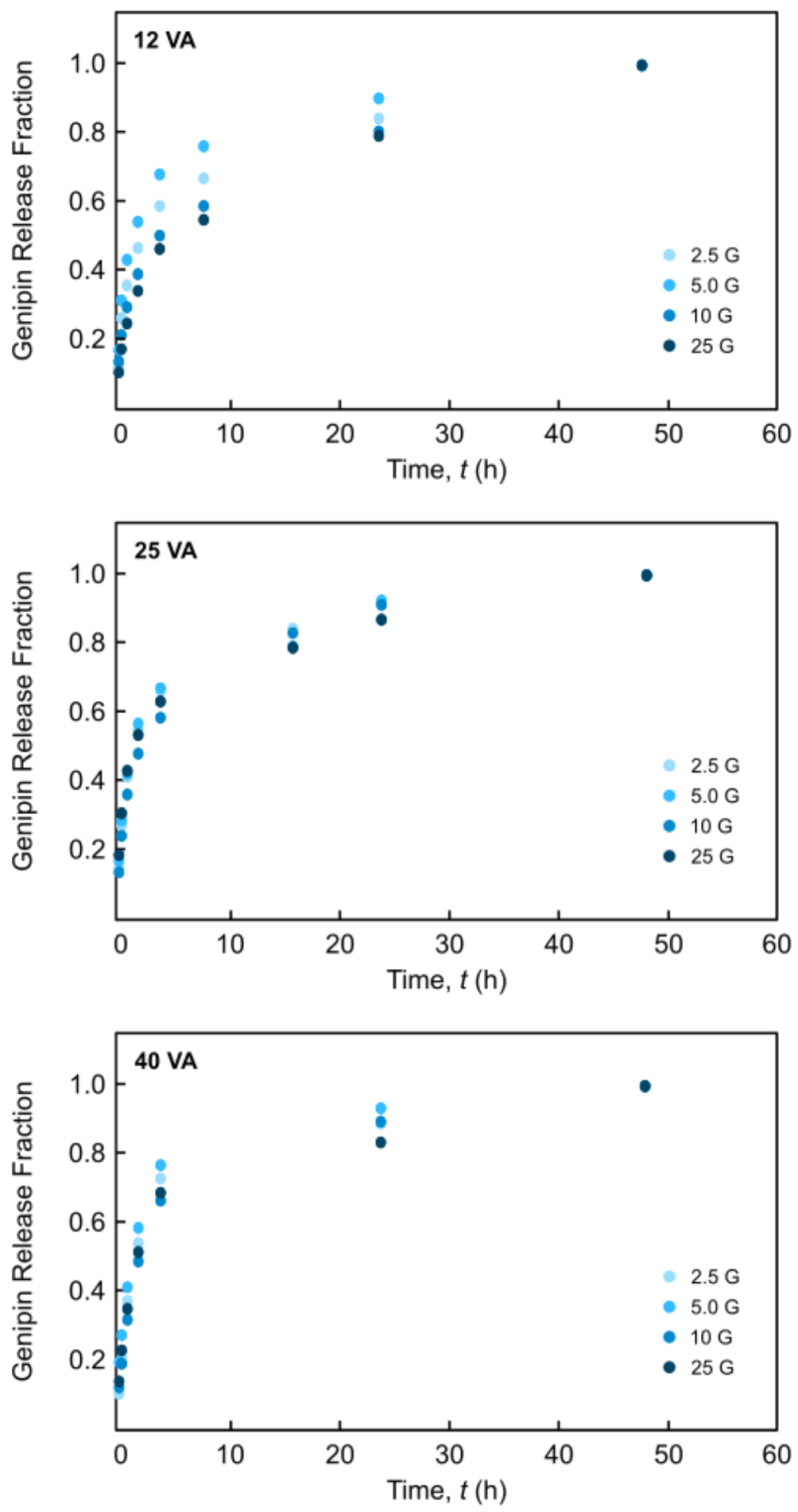

Figure S5. $m_{\text {genipin-PEVA,t }} / m_{\text {genipin-PEVA, } \infty}$ curves of genipin release from PEVA fibers loaded with $2.5,5,10$, and $25 \mathrm{wt} \%$ genipin and vinyl acetate concentrations of (a) $12 \mathrm{~mol} \% \mathrm{VA}$, (b) $25 \mathrm{~mol} \%$ $\mathrm{VA}$, and (c) $40 \mathrm{~mol} \%$. 


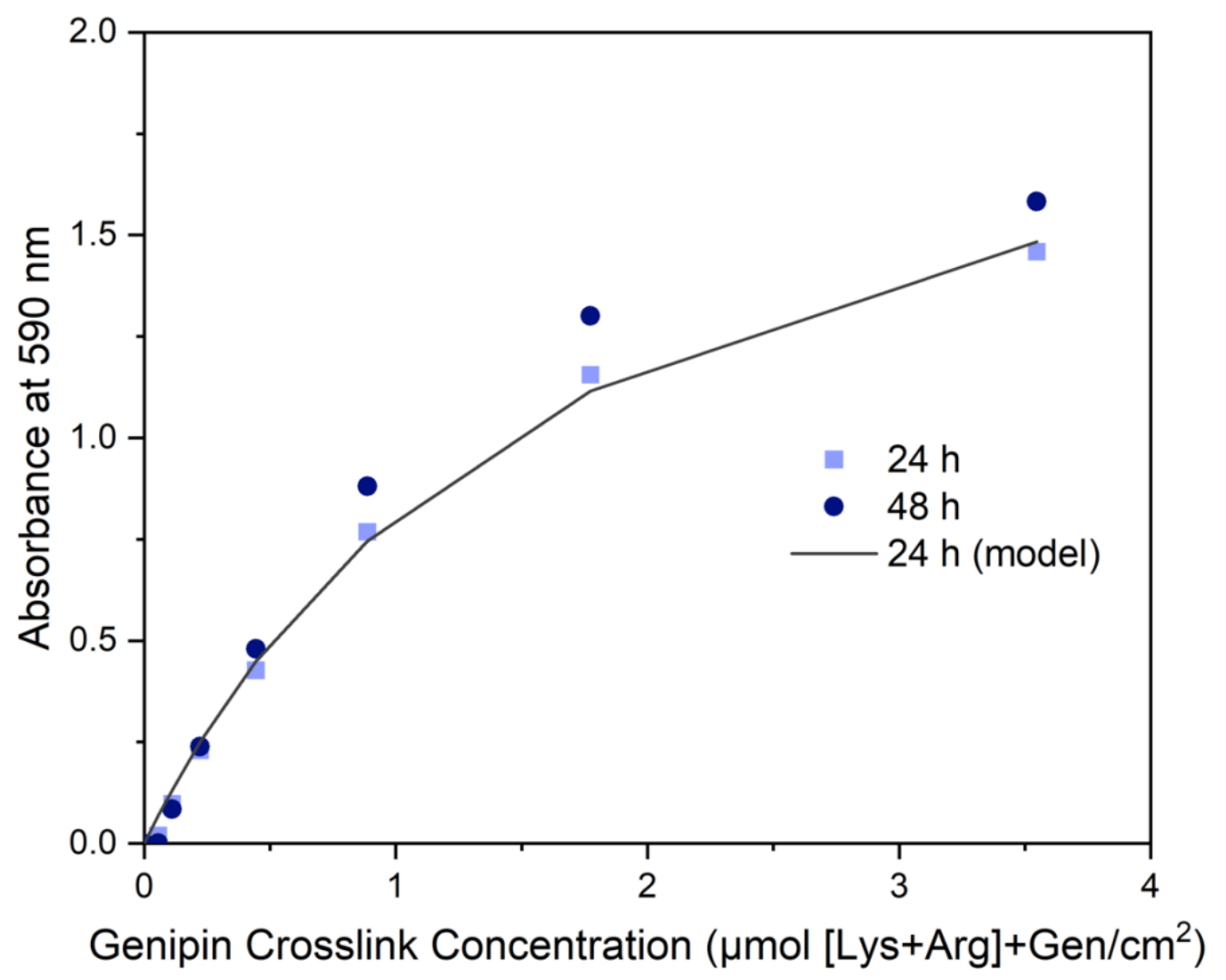

Figure S6. Plate reader standard curve of genipin crosslink concentration versus absorbance at $\lambda_{\text {Abs }}=590 \mathrm{~nm}$ after 24 hours $(\square)$ and 48 hours $(\bullet)$.

Figure S7. See video file. Time-lapse video of genipin diffusion from genipin-PEVA fiber (25 mol\% vinyl acetate, $25 \mathrm{wt} \%$ genipin) in an in vitro aneurysm flow model (6 hours). 